\title{
Pneumatosis cystoides intestinalis associated with pyloric stenosis
}

\author{
A. PADWELL* \\ F.R.C.S. \\ C. KOPELMAN $\dagger$ \\ M.D. \\ *Department of Surgery ' $B$ ', and $\dagger$ Department of Pathology, Safed Government Hospital, Israel
}

\begin{abstract}
Summary
Pneumatosis cystoides intestinalis is a rare condition characterized by the presence of gas-filled cysts in the bowel wall. A case is reported which was associated with peptic ulcer and in which there was involvement of the ileum and pre-pyloric region of the stomach. Stomach involvement is a rare occurrence. Chronic pneumoperitoneum was also present. The mechanism of its occurrence is discussed.
\end{abstract}

\section{Introduction}

Pneumatosis cystoides intestinalis is said to be either primary or secondary depending upon the presence of associated pathology. Koss (1952) reviewed 213 cases and found primary pneumatosis in $15.4 \%$. This group tended to show ileo-caecal involvement with sub-mucosal cysts. Stomach involvement was rare; $58 \%$ of the cases were associated with stenotic lesions of the pyloric area, in which mainly the small bowel was the site of cyst formation. Smith and Welter (1967) reviewed 33 cases and found small bowel involvement in $52 \%$; stomach involvement occurred in $5 \%$.

The authors report a case associated with peptic ulcer in which there was gross involvement of the ileum, along with the histological finding of submucous gas spaces in the stomach. Chronic pneumoperitoneum was also present.

\section{Case report}

A 37-year-old man who had been known to suffer from a peptic ulcer for 6 years was admitted with pyloric stenosis. Pneumoperitoneum had been noted on X-ray 3 years previously when he was admitted elsewhere for an acute exacerbation of pain. He refused surgery and was treated conservatively following which he was lost to follow-up until he presented to the authors' department with the complaints of progressive weight loss and vomiting after food.

On examination there was a marked succussion splash and evidence of recent weight loss, but no signs of an acute abdomen. On naso-gastric aspiration, 700 c.c. of fluid were obtained. Plain X-rays showed pneumoperitoneum, along with radiolucent cysts in a loop of bowel interposed between the diaphragm and the liver-the so-called Chilaiditi sign (Fig. 1). Subsequent gastroscopy and barium meal revealed pyloric stenosis with a pre-pyloric ulcer.

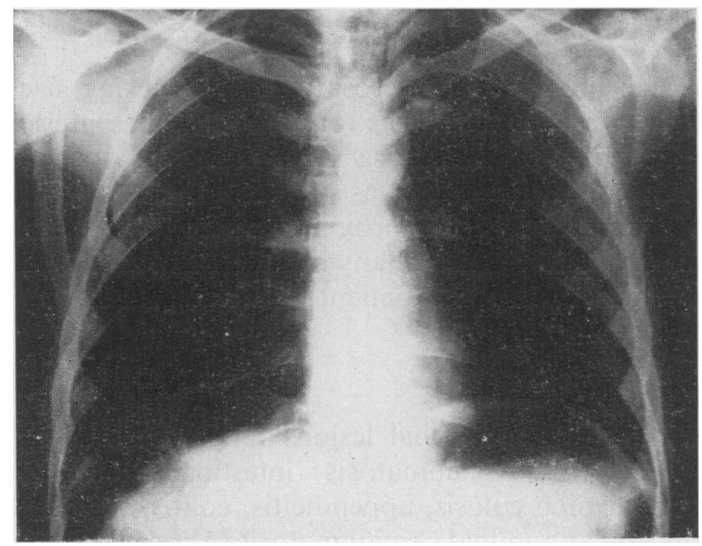

FIG. 1. Chilaiditi sign showing translucent cysts on interposed loop of bowel between liver and diaphragm.

At laparotomy, the pre-operative diagnosis of pyloric stenosis due to ulcer was confirmed. There was no evidence of perforation. A small amount of pink myxomatous gelatinous material was found in the peritoneal cavity. A segment of ileum, approximately $30 \mathrm{~cm}$ in length and ending about $10 \mathrm{~cm}$ from the ileo-caecal junction, was seen to be covered with multiple subserosal gas-filled cysts. Truncal vagotomy and antrectomy were performed and the diseased segment of ileum resected with end-to-end anastomosis.

Histological examination of the gastric antrum revealed a chronic pre-pyloric ulcer $12 \mathrm{~mm}$ in diameter extending into the muscularis. Several submucosal air spaces were seen adjacent to the ulcer (Fig. 2). Examination of the resected ileum 


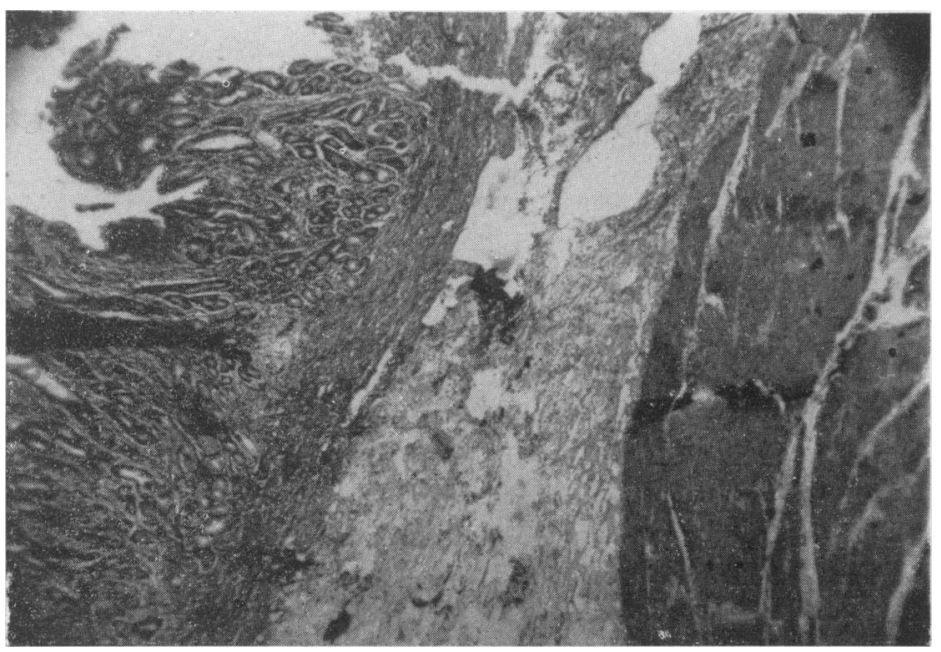

FIG. 2. Section of pylorus showing submucosal air spaces in region of ulcer.

revealed numerous subserosal cysts of varying size filled with odourless gas on the anti-mesenteric border. In the submucosa were many air spaces. The mucosa was intact. Gas spaces were also seen in the submucosa of the proximal resection margin. There was no evidence of any inflammatory reaction.

Postoperatively the patient made a satisfactory recovery.

\section{Discussion}

Other gastro-intestinal lesions reported as being involved with pneumatosis intestinalis include intestinal tuberculosis, appendicitis, colitis, regional ileitis and abdominal carcinomatosis (Thorpe, 1965). Marshak, Blum and Eliasoph (1956) reported the condition involving the left colon following sigmoidoscopy.

In Smith and Welter's (1967) review of 33 cases, 10 were infants below the age of one year. In these, the lesion was associated with severe fulminating colitis which pursued a fatal course.

The other group of patients in whom pneumatosis intestinalis is an associated finding is that suffering from chronic obstructive airways disease (Hughes et al., 1966; Doub and Shea, 1960). In contrast to those with pyloric obstruction, the pulmonary patients tend to show a preponderance of colonic lesions; a number of rectal lesions have also been reported (Wyatt, 1972).

A familial association has been reported by Underwood, Finnis and Scott (1978) who observed the condition in a mother and son. Pneumatosis coli has been described as a drug reaction to practolol (Thein and Asquith, 1977). Four cases were reported by Tsega (1975) in which the condition was as- $\overbrace{\varnothing}^{\infty}$ sociated with unexplained ascites.

The pathophysiology of the condition is not certain, but a mechanical theory seems to be the most likely explanation in those patients wi associated bowel lesions. It is postulated that a tear. in the bowel mucosa results in the liberation of gas into the bowel wall. The gas is further distributed by peristalsis. Smith and Welter (1967) believe that gas under pressure is a pre-requisite for this theory to $\approx$ apply, as might occur in obstructive bowel lesions. $\stackrel{\perp}{\perp}$ The presence of gas spaces in the submucosa of the $\overrightarrow{\vec{B}}$ pyloric region adjacent to the ulcer in the present $\frac{0}{3}$ patient is in keeping with this theory, as is the further finding of submucosal air spaces in the proximal resection margin of the ileum. Distal to this level, the gas must have broken through the muscularis to 3 . produce the larger subserosal cysts. The finding of $\dot{\sigma}$ submucosal gas spaces in the region of the ulcer has not previously been reported.

The pneumoperitoneum presumably arose as a result of rupture of one or more of the subserosal 응 ileal cysts, or even possibly from the ulcer itself. It $>$ has been shown that gas, without fluid, can leak into the peritoneum causing a pneumoperitoneum $N$ without a concomitant inflammatory reaction or peritonitis (Hall, 1971). Tension pneumoperitoneum $\stackrel{N}{O}$ has been recorded; and this may have been the cause $\underset{\omega}{N}$ of the patient's acute pain 3 years earlier when there was then a finding of pneumoperitoneum on X-ray 0 but no evidence of a previous perforation was found at laparotomy. This is presumably a dynamic $\stackrel{\odot}{+}$ process, air being liberated into the peritoneal cavity $T$ and then re-absorbed, only to be further liberated $\underset{\mathbb{D}}{\stackrel{0}{0}}$ 
and the process repeating itself thus explaining a chronic pneumoperitoneum of at least 3 years' duration.

The explanation put forward for the cases associated with pulmonary disease is that rupture of emphysematous bullae occurs causing a pneumomediastinum. Air then enters the sheaths of the pulmonary arteries which are in continuity with that of the aorta and its branches. The air thus passes down retroperitoneally and along the mesenteric vessels reaching the bowel wall (Keyting et al., 1961). It is, however, difficult to reconcile the formation of submucous bowel cysts with this theory, although not subserosal ones.

A rival theory implicates intestinal bacteria as the source of the gas. Yale, Balish and Wu (1974) reproduced the condition in germ-free rats by introducing cultures of Clostridium perfringens. Chemical analysis of the cysts (Forgacs, Wright and Wyatt, 1973) has shown the gas content to be up to $50 \%$ hydrogen, and raised fasting breath hydrogen levels have been found (Gillon et al., 1979a) supporting the bacterial theory.

Because of this gas content of the cysts, it has been postulated that they can be deflated if the total pressure of the gases in venous blood can be lowered by breathing a high oxygen concentration, which alters the balance of diffusion in favour of absorption (Forgacs et al., 1973). This has been shown to be an effective method of treatment (Wyatt, 1975; Down and Castledon, 1975). However, the reappearance of the cysts has been found to occur after the cessation of the oxygen therapy (Van der Lynden, 1974).

A high end-expiratory hydrogen fasting value has also been confirmed (Van der Lynden and Marsell, 1979), and this was then reduced by feeding their patients an elemental diet, i.e. one absorbed in the small gut. Following this, the hydrogen values fell and the cysts regressed, suggesting that when colonic gas production is reduced the cysts regress. Should intestinal gas production increase one might then expect cyst formation to occur: this has been found after jejuno-ileal bypass surgery when there may be an increase in carbohydrate delivered to the bowel flora for fermentation (Feinberg et al., 1977; Passaro, Drenick and Wilson, 1976; Sicard, Vaughan and Wise, 1976). Co-existent pulmonary disease may then affect the excretion of hydrogen resulting in its decreased absorption and accumulation at its site of production. Antiobiotics, metronidazole (Ellis, 1980) and ampicillin (Holt et al., 1978), have also been used with success in the treatment of colonic lesions, again supporting an infective aetiology.

However, the evidence pointing to a bacterial aetiology is in those cases of pneumatosis cystoides affecting the large bowel, often in association with cardiopulmonary disease. The present patient was not of this type: his lesions were confined to the small bowel and gas spaces were seen adjacent to the ulcer and in the proximal ileal resection margin. It is felt that the mechanical theory is still the most likely explanation in this patient.

The clinical course of the condition is generally assumed to be benign and self-limiting. It may be an incidental finding at laparotomy for another condition. Many authors recommend no treatment apart from that directed towards the primary disorder (Gillon, Holt and Sircus, 1979b). Because of the chronic pneumoperitoneum, with the risk of tension pneumoperitoneum, and the large segment of bowel involved it was decided in the present case to resect the involved segment.

Complications sometimes requiring surgical intervention have been reported, such as obstruction, volvulus (Jones, 1948) and bleeding. Other sequelae include diarrhoea and malabsorption (Yunick and Fradkin, 1958).

\section{Acknowledgments}

We would like to thank Dr G. Canaan, Chief of Surgery B, and Dr B. Scolnick, Chief of Pathology, Safed Government Hospital, for their assistance in producing this paper.

\section{References}

Doub, H.P. \& SheA, J.J. (1960) Pneumatosis cystoides intestinalis. Journal of the American Medical Association, 172, 1238.

Down, R.H.L. \& Castledon, W.M. (1975) Oxygen therapy for pneumatosis coli. British Medical Journal, 1, 493.

Ellis, B.W. (1980) Symptomatic treatment of primary pneumatosis coli with metronidazole. British Medical Journal, 280, 763.

Feinberg, S.B., Schwartz, M.Z., Clifford, S., Buchwald, H. \& VARCO, R.L. (1977) Significance of pneumatosis cystoides intestinalis after jejunoileal bypass. American Journal of Surgery, 133, 149.

Forgacs, P., Wright, P.H. \& Wyatt, A.P. (1973) Treatment of intestinal gas cysts by oxygen breathing. Lancet, $\mathbf{i}, 579$.

Gillon, J., Holt, S. \& Sircus, W. (1979b) Pneumatosis coli and sigmoid volvolus: a report of four cases. British Journal of Surgery, 66, 802.

Gillon, J., TAdesse, K., Logan, R.F.A., Holt, S. \& Sircus, W. (1979a) Breath hydrogen in pneumatosis cystoides intestinalis. Gut, 20, 1008.

HaLl, R. (1971) Delayed tension pneumoperitoneum after colostomy closure. British Journal of Surgery, 58, 574.

Holt, S., Stewart, I.C., Heading, R.C. \& Macpherson, A.I.S. (1978) Resolution of primary pneumatosis coli. Journal of the Royal College of Surgeons of Edinburgh, 23, 297.

Hughes, D.T.D., Gordon, K.C.D., Swann, J.C. \& Bolt, G.L. (1966) Pneumatosis cystoides intestinalis. Gut, 7, 553.

JONES, J.D.T. (1948) Gas cysts of the intestine. British Journal of Surgery, 36, 49.

Keyting, W.S., McCarver, R.R., Kovarik, J.L. \& DayWITT, A.L. (1961) Pneumatosis intestinalis: a new concept. Radiology, 76, 733.

Koss, L.G. (1952) Abdominal gas cysts (pneumatosis cystoides intestinorum hominis): an analysis with a report of a case and a critical review of the literature. Archives of Pathology, 53, 523. 
MarshaK, R.H., Blum, S.D. \& Eliasoph, J. (1956) Pneumatosis involving the left side of the colon. Journal of the American Medical Association, 161, 1626.

Passaro, E., Drenick, E. \& Wilson, S.E. (1976) Bypass enteritis: a new complication of jejunoileal bypass for obesity. American Journal of Surgery, 131, 169.

SiCARD, G.A., Vaughan, R. \& Wise, L. (1976) Pneumatosis cystoides intestinalis: an unusual complication of jejunoileal bypass. Surgery, 79, 480.

Smith, B.H. \& Welter, L.H. (1967) Pneumatosis intestinalis. American Journal of Clinical Pathology, 48, 455.

Thein, S.L. \& Asquith, P. (1977) Pneumatosis coli, a complication of practolol. British Medical Journal, 1, 268.

ThORPE, G.J. (1965) Pneumatosis cystoides intestinalis associated with abdominal carcinomatosis. Postgraduate Medical Journal, 41, 41.

TsegA, E. (1975) Pneumatosis cystoides intestinalis with unexplained ascites in Ethiopian patients. British Journal of Surgery, 62, 379.

Underwood, J.W., Finnis, D. \& ScotT, W. (1978) Pneuma- tosis coli: a familial association. British Journal of Surger 65, 64-65.

VAN DER LYNDEN, W. (1974) Reappearance of intestinal gर्ds cysts after oxygen therapy. Lancet, ii, 1389.

VAN DeR LyNDen, W. \& MARsell, R. (1979) Pneumatos cystoides coli associated with high hydrogen excretion? treatment with an elemental diet. Scandinavian Journal at Gastroenterology, 14, 173.

WyatT, A.P. (1972) Pneumatosis cystoides intestinali Proceedings of the Royal Society of Medicine, 65, 780.

WyatT, A.P. (1975) Prolonged symptomatic and radiç logical remission of colonic gas cysts after oxygen therapy. British Journal of Surgery, 62, 837.

YAle, C.E., BALISH, E. \& WU, J.P. (1974) The bacteriat etiology of pneumatosis cystoides intestinalis. Archives $\delta \mathcal{S}^{\circ}$ Surgery, 109, 89.

Yunick, A.M. \& Fradkin, N.F. (1958) Fatal sprue (ma absorption) syndrome secondary to extensive pneumatos? cystoides intestinalis. Gastroenterology, 35, 212. 